\title{
Effects of Rehabilitation System with Efficient Vision-based Action Identification Techniques on Physical Fitness Promotion in Patients with Schizophrenia
}

\author{
Yen-Lin Chen, ${ }^{1}$ Chin-Hsuan Liu, ${ }^{1}$ Chao-Wei Yu, ${ }^{1}$ and Posen Lee ${ }^{2 *}$ \\ ${ }^{1}$ Department of Computer Science and Information Engineering, National Taipei University of Technology, \\ 1, Sec. 3, Zhongxiao E. Rd. Taipei 10608, Taiwan \\ ${ }^{2}$ Department of Occupational Therapy, I-Shou University, \\ No. 8, Yida Rd., Jiaosu Village, Yanchao District, Kaohsiung 82445, Taiwan
}

(Received March 28, 2018; accepted October 18, 2018)

Keywords: rehabilitation system, depth sensors, action identification techniques, schizophrenia, physical fitness

Nowadays, there are many human pose estimation systems that extract skeletons or skeleton points from depth sensors. These action identification systems have been developed for somatosensory games for entertainment use, and some of these have been used for medical purposes. Most of these have proven that the action identification technology is practical, but its medical use has not been confirmed. Therefore, besides the general treatment of mental illness, we tried to add a rehabilitation program to promote physical fitness based on a visionbased action sensor system. It is expected to effectively improve the physical functions of patients with mental illness. There are some somatosensory games that claim that they can achieve the effects of sports and entertainment, and some of these have been used for the physical training and physical rehabilitation, but none of them have been used for the physical rehabilitation of patients with schizophrenia. Thus, the purpose of this study was to examine the effects of a rehabilitation system with efficient vision-based action identification techniques on physical fitness promotion in patients with schizophrenia through the sequential-withdrawal design of a single subject research. Three patients with schizophrenia were recruited. Each subject received examinations for the 6-Minute Walk Test (6MWT) and Timed Up and Go Test (TUG) in each period, and received physical training with a rehabilitation system with an efficient vision-based action identification technique during the treatment phase. The results of this study were as follows. First, the rehabilitation system was easy to operate and use, even for people with mental illness. Second, compared with traditional physical exercise, patients with schizophrenia are more willing to accept physical fitness training in the form of somatosensory games rather than traditional treatments. Third, the rehabilitation system with efficient visionbased action identification techniques is suitable for physical promotion programs for patients with schizophrenia. Fourth, the program in the rehabilitation system helps improve the physical performance of people with schizophrenia. Fifth, the system keeps a record of the rehabilitation processing and physical status as a treatment document of patients with schizophrenia.

*Corresponding author: e-mail: posenlee@isu.edu.tw https://doi.org/10.18494/SAM.2018.2131 


\section{Introduction}

Nowadays, there are many human pose estimation systems that extract skeletons or skeleton points from depth sensors. ${ }^{(1-6)}$ Although these pose estimation systems have been developed and presented, most of those existing systems mainly focus on providing human pose estimation. Some of them are used for entertainment games, some are used to operate devices with poses or gestures, and some are used for security monitoring. Some makers of somatosensory games claim that the games can achieve the effects of sports and entertainment, and some of these have been used for physical exercise. ${ }^{(7-9)}$ However, these somatosensory games are not designed for medical use and lack clinical evidence.

Patients with schizophrenia often have problems such as obesity and poor cardiorespiratory capacity. ${ }^{(10)}$ Many studies have shown relevant benefits of physical fitness conditioning for patients with mental illness, and many studies have clearly pointed out that through exercise, the negative symptoms of patients with schizophrenia can be significantly improved. ${ }^{(11)}$ The rehabilitation of patients with schizophrenia not only reduces their psychiatric symptoms, but also improves their physical functions. Thus, general psychiatric treatment combined with physical fitness training is the best treatment plan for patients with schizophrenia. ${ }^{(12)}$

In rehabilitation, a patient receives treatment to improve impaired mobility or increase functional capability. Treatment typically takes a long time to achieve a positive effect, and if the treatment is stopped, the reversal of progress may occur. ${ }^{(13,14)}$ Therefore, rehabilitation is a very lengthy process that takes a heavy psychological, physical, and economic toll on patients, and might make patients drop out from courses of treatment. ${ }^{(15)}$ Home-care systems and telemedicine have become the trend because of the integration of technology into the practice of medicine. ${ }^{(16-20)}$ A home program of rehabilitation can also make rehabilitation programs easier to implement and enable more frequent exercise.

In this study, we propose a rehabilitation system ${ }^{(21)}$ and aim to examine the effects of the rehabilitation system with efficient vision-based action identification techniques on physical fitness promotion in patients with schizophrenia. Accordingly, the rehabilitation system for physical fitness promotion in patients with schizophrenia will be examined from the following viewpoints: (1) Is this system easy to operate even for patients with mental illness? (2) Does the system make the patients become interested in and willing to accept physical fitness training? (3) Is the system usable and suitable for patients with schizophrenia? (4) Is the system helpful in physical fitness training for patients with schizophrenia? (5) Does the system record and summarize the training process and physical status data as a treatment document?

\section{Materials and Methods}

In this study, the major materials used were programs of the rehabilitation system with efficient vision-based action identification techniques. The sequential-withdrawal design of a single subject research was adopted. ${ }^{(22)}$ Each subject received physical fitness training with the rehabilitation system during the intervention phase and physical fitness measurements to monitor the effects of physical training and changes in physical fitness in each phase. 
Subjects were recruited from a clinical psychiatric center and gave informed consent prior to participation. We adopted the Mini-Mental State Examination (MMSE) as a screen test, and subjects were included in this study if they obtained a score above $15 .{ }^{(23)}$ We included three participants in this study, which was conducted for three months.

\subsection{Procedure}

The sequential-withdrawal design of a single-subject research was adopted. ${ }^{(22)}$ The physical condition of the participants was observed and assessed, and there was no intervention during the baseline phase for three consecutive days. The baseline phase establishes a benchmark against which participants' physical fitness in subsequent conditions can be compared. The next three days was alloted for the intervention phase, where the participants received evaluation and one hour of physical fitness training with the proposed system each day. The seventh to the ninth day was the first withdrawal phase, where the participants received evaluation and physical fitness training without the proposed system. The last phase was the second withdrawal phase, wherein the participants received evaluation and no intervention during this period. Figure 1 shows the diagram of this study.

\subsection{Subjects}

The subjects were recruited from a clinical psychiatric center and gave informed consent prior to participation. The subjects with the following characteristics were included: Diagnosed with schizophrenia on the basis of the Diagnostic and Statistical Manual of Mental Disorders,

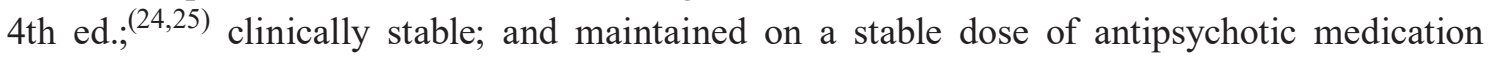
for at least 1 month prior to the research testing sessions. All the patients were offered the

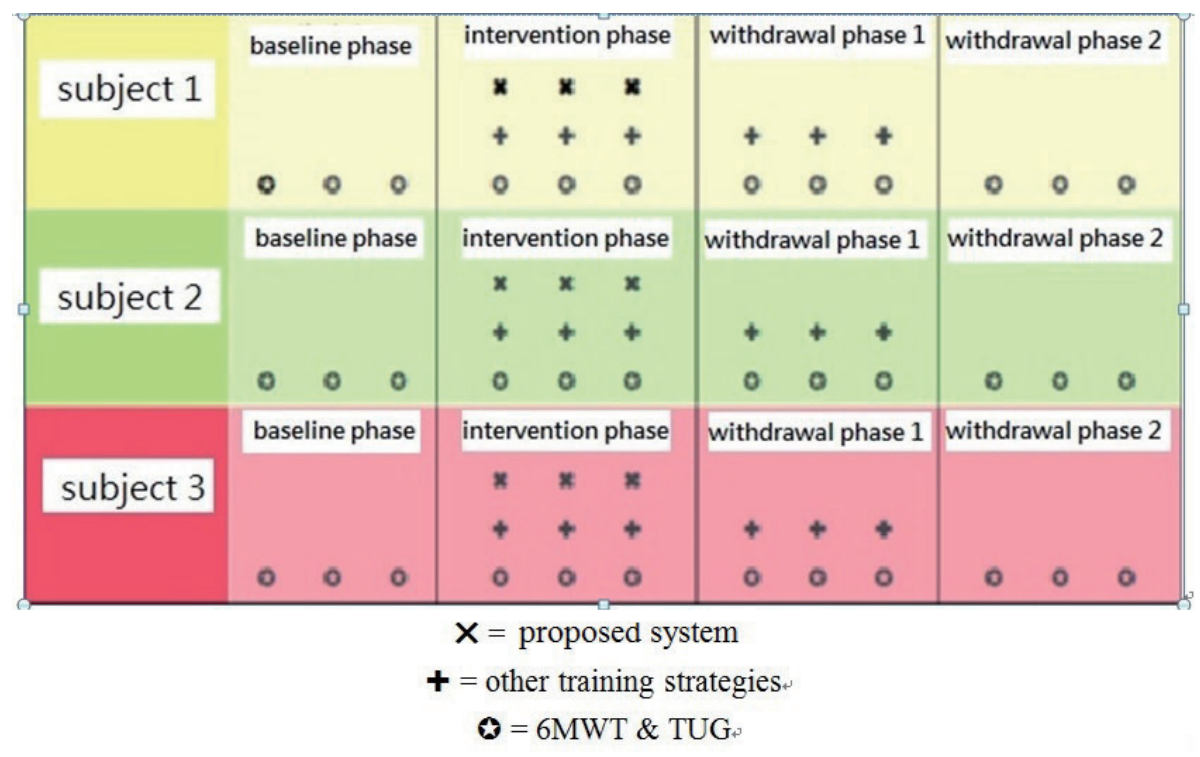

Fig. 1. (Color online) Diagram of this study. 
opportunity to join the study during a visit to the clinic and all gave written informed consent before participating in the study. Patients were excluded from the study (a) if they were medically unstable, had other major diseases, or had secondary diagnoses of mental retardation, dementia, or neurological impairment or were suffering an episode of major depression, (b) if they were suffering from a severe medical or neurological condition or another psychiatric disorder that required treatment, (c) if they were participating in a clinical trial, and/or (d) if they could not follow instructions or their MMSE scores were below $15 .^{(23)}$ The study protocol was reviewed and approved by the Institutional Review Board of the study hospital.

\subsection{Materials}

The use of the rehabilitation system with efficient vision-based action identification techniques $^{(21)}$ is as follows: We assume that the user is sitting or standing about $80-100 \mathrm{~cm}$ in front of the depth sensor to use this system. The proposed system can identify human skeleton and estimate human pose in sitting and standing positions. This system can be connected to audio-visual equipment at home, which provides a familiar and convenient environment. Figure 2 shows the experimental setup used in this study. Table 1 shows the hardware and software of the proposed system.

The rehabilitation system captured the user's movements through a Kinect camera and presented them on a screen. Then, the image of the user's motion combined with the builtin designed game in the system was outputed on the screen at the same time. The training programs included seven types of rehabilitation movement for the training of specific muscle groups in this rehabilitation system. The content of the training was that a red dot appears at

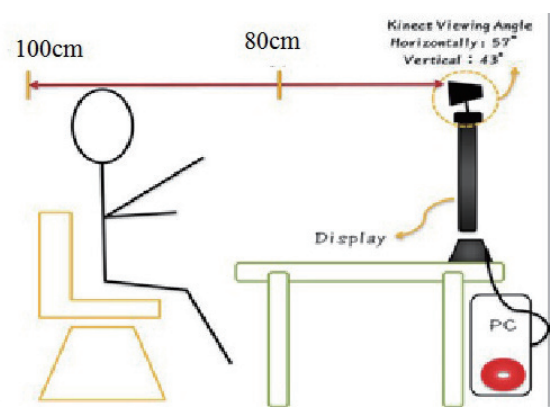

Fig. 2. (Color online) Experimental setup used in this study.

Table 1

Hardware and software of the proposed system.

\begin{tabular}{cc}
\hline Hardware & Software \\
\hline CPU: Intel Core(TM)2 Quad 2.33GHz 2.34GHz & OS: WIN7 \\
RAM: 4 GB & Platform: QT 2.4.1 \\
Depth sensor: Kinect & Library: QT 4.7.4, OpenCV-2.4.3 \\
& OpenNI1.5.2.23 (only for RGB image and depth \\
RGB camera: LogitechC920 & information) \\
\hline
\end{tabular}


the center of the screen to guide the user to the correct starting position. After that, the red dot will appear elsewhere to induce the user to make the correct movement. The screen presented the desired movement and the user's movement at the same time. The user could imitate the demonstration and compare the movement differences on the screen. Table 2 shows the experimental training programs in this study.

\subsection{Measures of physical function}

To monitor outcomes of physical fitness training with the rehabilitation system and changes in physical fitness over time, we used the 6-Minute Walk Test (6MWT) and Timed Up and Go Test (TUG) to measure physical functions to compare changes during each phase.

\subsubsection{MWT}

The 6MWT is safer, easier to administer, better tolerated, and better reflects activities of daily living than other walk tests. The 6WMT is a submaximal exercise test used to assess aerobic capacity and endurance. The distance covered over a time of $6 \mathrm{~min}$ is used as the outcome with which changes in performance capacity are compared. ${ }^{(26)}$ When conducting the 6MWT, do not walk with the patient or assist the patient in carrying or pulling his or her supplemental oxygen. Do not use a treadmill on which the patient adjusts the speed and/or the slope. Do not use an oval or circular track. Use standardized phrases while speaking to the patient. ${ }^{(27)}$

\subsubsection{TUG}

The TUG is a simple, quick, and widely used clinical performance-based measure of lower extremity function, mobility, and fall risk. ${ }^{(28)}$ The area for the TUG test was set up by measuring $3 \mathrm{~m}$ from the front legs of a straight-backed armchair. The subject was instructed to "Sit with your back against the chair and your arms on the arm rests. On the word 'go', stand upright, then walk at your normal pace to the line on the floor, turn around, return to the chair, and sit down". The stopwatch was started on the word 'go' and stopped when the subject returned to the starting position. ${ }^{(29)}$ The subject was observed and timed while he rose from an arm chair, walked $3 \mathrm{~m}$, turned, walked back, and sat down again. ${ }^{(30)}$

Table 2

Experimental training programs: seven types of rehabilitation movement.

\begin{tabular}{|c|c|c|c|c|c|c|c|}
\hline $\begin{array}{l}\text { Position } \\
\text { No. }\end{array}$ & $\begin{array}{l}\text { Position } 0 \\
\text { (initial) }\end{array}$ & Position 1 & Position 2 & Position 3 & Position 4 & Position 5 & Position 6 \\
\hline $\begin{array}{l}\text { Position of } \\
\text { skeleton } \\
\text { points }\end{array}$ & & & 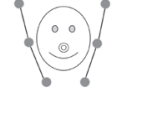 & & 0 & & \\
\hline
\end{tabular}




\subsection{Data analyses}

The effects of the rehabilitation system with efficient vision-based action identification techniques on physical fitness promotion in patients with schizophrenia were evaluated on the basis of the 6MWT and TUG tests. The analysis was based on visual comparison between the phases. Data were analyzed by visual analysis.

\section{Results}

We recruited three participants, all of whom completed physical fitness training programs and assessments of physical functions. No one indicated physical or mental discomfort caused by physical training through the rehabilitation system. Through clinical observation, it was found that, using the rehabilitation system with efficient vision-based action identification techniques to provide visual clues and image feedback for rehabilitation, did not induce symptoms such as hallucinations or delusions.

During the baseline phase, the mean scores of $6 \mathrm{MWT}$ and TUG showed that the physical conditions of the three subjects were very different. The scores of the three subjects in the fourth session were lower than those in the first three sessions. Figure 3 shows the chart of the results of the 6MWT and TUG of the three subjects.
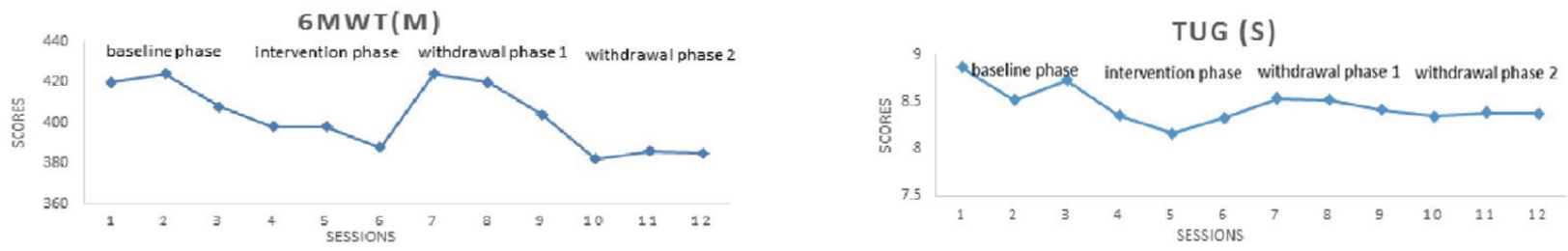

(a)
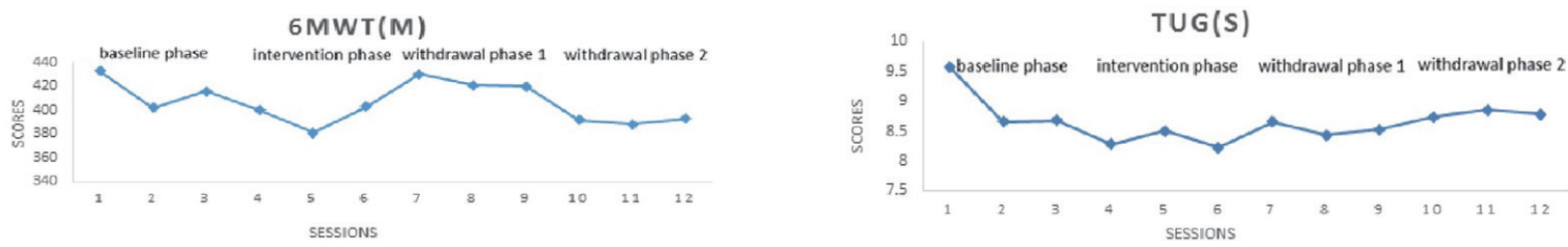

(b)
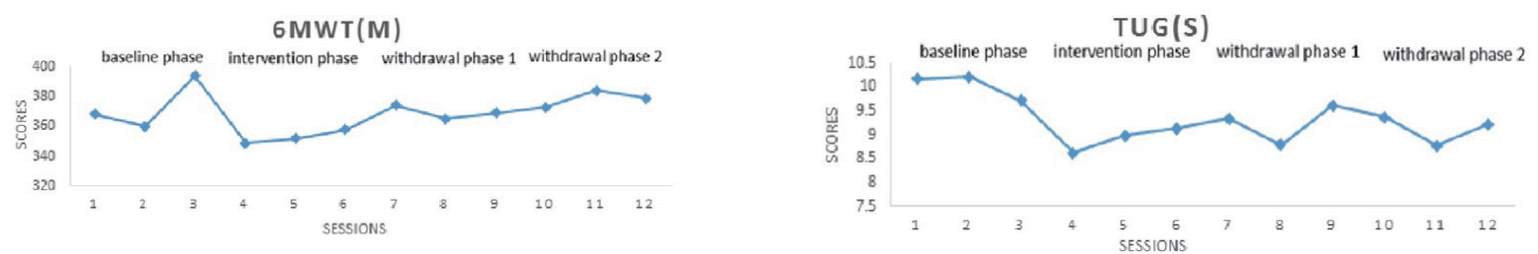

(c)

Fig. 3. (Color online) Chart of results of 6MWT and TUG of three subjects. (a) Subject 1, (b) subject 2, and (c) subject 3 . 
For both 6MWT and TUG, the scores of the first to the third phase of the first subject showed a downward trend; the slopes of the baseline and interventional phases were similar, but there was a more significant decline during the first withdrawal phase. Compared with the other phases, the slope was slightly upward only in the second withdrawal phase. The variability of the trend of the interphase comparison was also similar; the positive effect only appeared between withdrawal phases 1 and 2 . Table 3 shows the results of the analysis of the intra- and interphases of the $6 \mathrm{MWT}$ of subject 1 . Table 4 shows the results of the analysis of the intra- and interphase of the TUG of subject 1.

The scores of the 6MWT of the second subject showed a downward trend in the baseline and first withdrawal phases, but an upward trend in the intervention and second withdrawal phases. The interphase comparison showed that there was a negative effect between the second and third phases, and a positive effect between the baseline and intervention phases, and between the first and second withdrawal phases. The scores of TUG from the first to the third phase showed a downward trend, but an upward trend during the last phase. The variability of the trend of interphase comparison showed a positive effect between the first and second withdrawal phases. Table 5 shows the results of the analysis of the intra- and interphases of the $6 \mathrm{MWT}$ of subject 2. Table 6 shows the results of the analysis of the intra- and interphases of the TUG of subject 2 .

The scores of the 6MWT of the third subject showed an upward trend during the baseline, intervention, and second withdrawal phases, but a downward trend during the third phase. The interphase comparison showed that there was a negative effect between the second and third phases, and a positive effect between the first and second withdrawal phases. The scores of

Table 3

Results of the analysis of the intra- and interphases of the 6MWT of subject 1.

\begin{tabular}{lcccc}
\hline Intraphase & Baseline phase & Intervention phase & Withdrawal phase 1 & Withdrawal phase 2 \\
\hline Sessions of phase & 3 & 3 & 3 & 3 \\
Range of level & $408-424$ & $388-398$ & $404-424$ & $382-386$ \\
Change of phase & -12.00 & -10.00 & -20.00 & 3.00 \\
Means of level & 417.33 & 394.67 & 416.00 & 384.33 \\
Stability & $100.0 \%$ & $100.0 \%$ & $100.0 \%$ & $100.0 \%$ \\
Trend and & $\backslash$ & $\backslash$ & $\backslash$ & $/$ \\
data path of trend & $(-)$ & $(-)$ & $(-)$ & $(+)$ \\
Stability of trend & $100 \%$ & $100 \%$ & $100 \%$ & $100 \%$ \\
\hline
\end{tabular}

\begin{tabular}{lccc}
\hline Interphase & $\begin{array}{c}\text { Baseline phase/ } \\
\text { Intervention phase }\end{array}$ & $\begin{array}{c}\text { Intervention phase/ } \\
\text { Withdrawal phase } 1\end{array}$ & $\begin{array}{c}\text { Withdrawal phase 1/ } \\
\text { Withdrawal phase 2 }\end{array}$ \\
\hline Change between phases & -10.00 & 36.00 & -22.00 \\
Means between phases & -22.66 & 21.33 & -31.67 \\
Variability of trend and effect & $\|$ & $\|$ & $\checkmark$ \\
& None & None & Positive \\
Variability of trend stability & Stable to stable & Stable to stable & Stable to stable \\
Percentage of overlap & $100 \%$ & $0 \%$ & $100 \%$ \\
\hline
\end{tabular}


Table 4

Results of the analysis of the intra- and interphases of the TUG of subject 1.

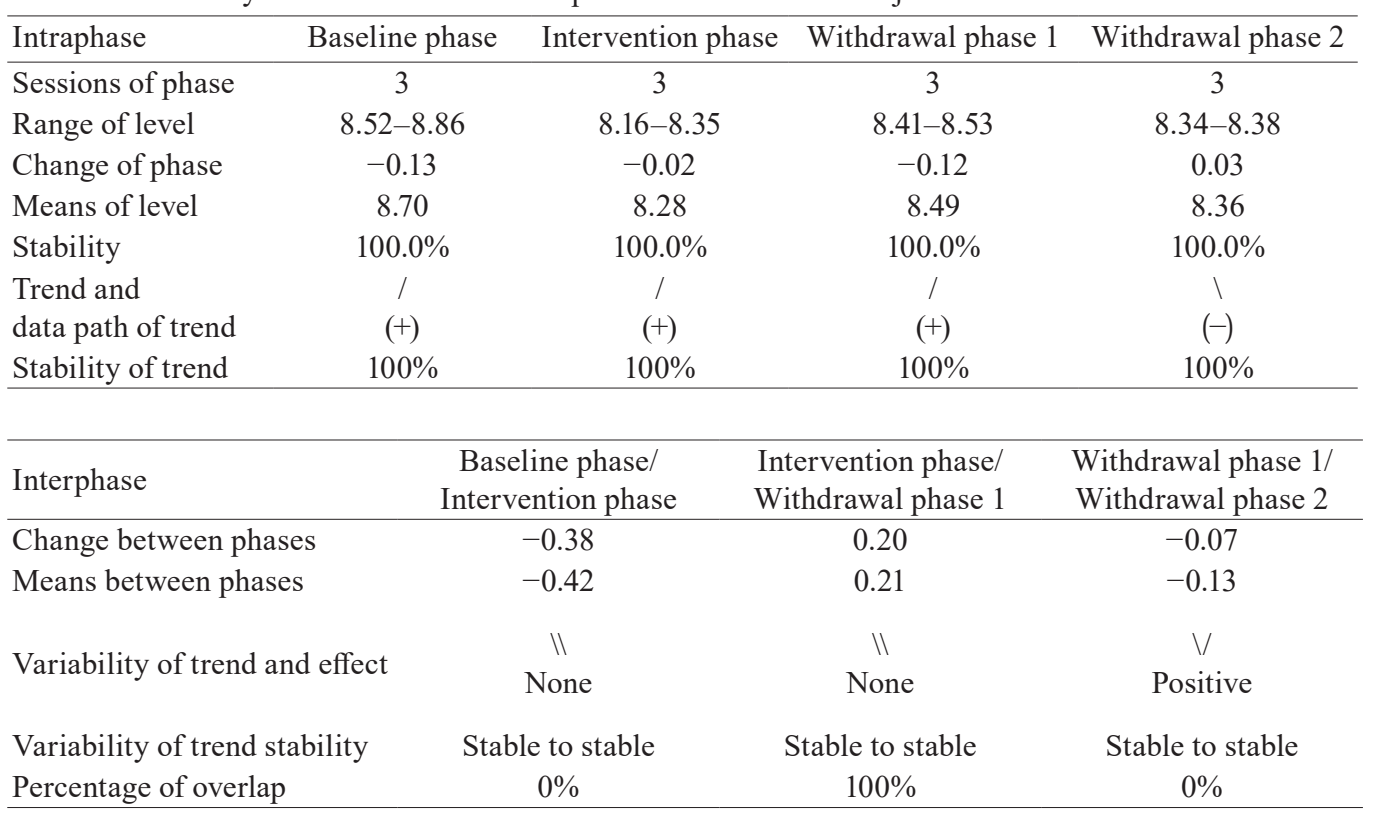

Table 5

Results of the analysis of the intra- and interphases of the 6MWT of subject 2.

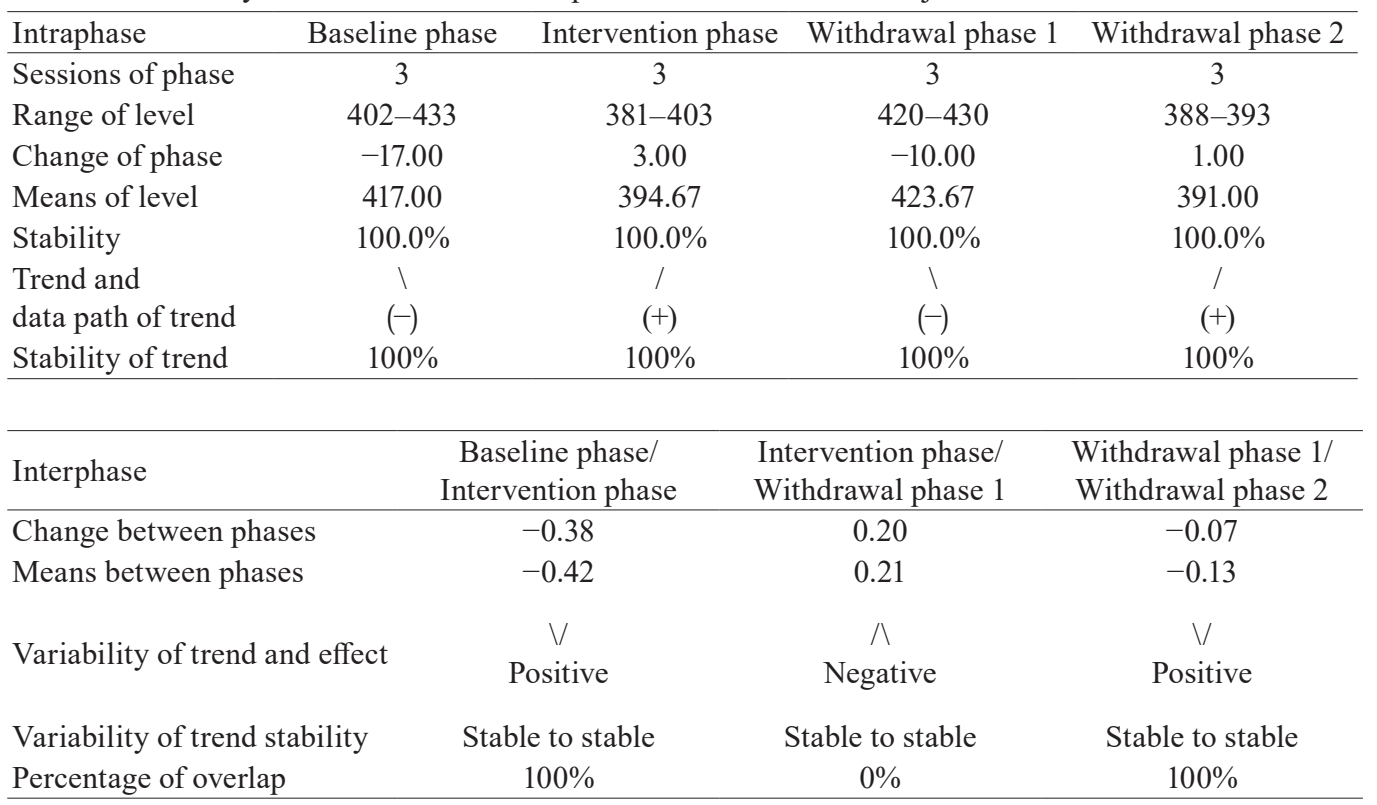

TUG showed a downward trend during the baseline and second withdrawal phases, but an upward trend during the second and third phases. The effect on the interphase was negative between the baseline and intervention phases, but positive between the first and second 
Table 6

Results of the analysis of the intra- and interphases of the TUG of subject 2.

\begin{tabular}{lcccc}
\hline Intraphase & Baseline phase & Intervention phase & Withdrawal phase 1 & Withdrawal phase 2 \\
\hline Sessions of phase & 3 & 3 & 3 & 3 \\
Range of level & $8.66-9.57$ & $8.23-8.50$ & $8.43-8.66$ & $8.74-8.86$ \\
Change of phase & -0.89 & -0.05 & -0.13 & 0.04 \\
Means of level & 8.97 & 8.34 & 8.54 & 8.79 \\
Stability & $66.67 \%$ & $100.0 \%$ & $100.0 \%$ & $100.0 \%$ \\
Trend and & $\backslash$ & $\backslash$ & $\backslash$ & $/$ \\
data path of trend & $(+)$ & $(+)$ & $(+)$ & $(-)$ \\
Stability of trend & $100 \%$ & $100 \%$ & $100 \%$ & $100 \%$ \\
\hline
\end{tabular}

\begin{tabular}{lccc}
\hline Interphase & $\begin{array}{c}\text { Baseline phase/ } \\
\text { Intervention phase }\end{array}$ & $\begin{array}{c}\text { Intervention phase/ } \\
\text { Withdrawal phase } 1\end{array}$ & $\begin{array}{c}\text { Withdrawal phase 1/ } \\
\text { Withdrawal phase 2 }\end{array}$ \\
\hline Change between phases & -0.4 & 0.43 & 0.21 \\
Means between phases & -0.63 & 0.2 & 0.25 \\
Variability of trend and effect & $\|$ & $\|$ & $V$ \\
Variability of trend stability & None & None & Positive \\
Percentage of overlap & $0 \%$ & Stable to stable & Stable to stable \\
\hline
\end{tabular}

Table 7

Results of the analysis of the intra- and interphases of the 6MWT of subject 3.

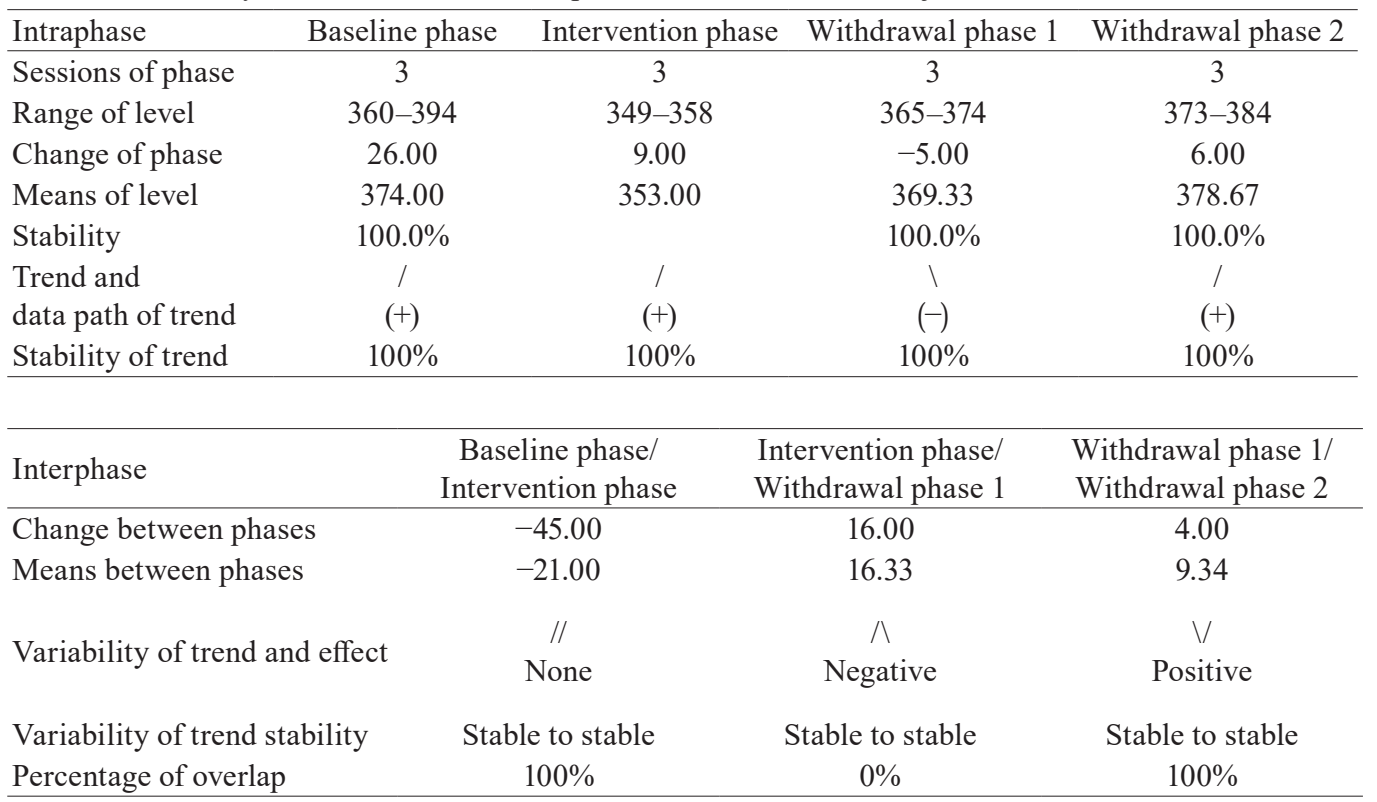

withdrawal phases. Table 7 shows the results of the analysis of the intra- and interphases of the $6 \mathrm{MWT}$ of subject 3 . Table 8 shows the results of the analysis of the intra- and interphases of the TUG of the subject 3 . 
Table 8

Results of the analysis of the intra- and interphases of the TUG of subject 3.

\begin{tabular}{|c|c|c|c|c|}
\hline Intraphase & Baseline phase & Intervention phase & e Withdrawal phase 1 & Withdrawal phase 2 \\
\hline Sessions of phase & 3 & 3 & 3 & 3 \\
\hline Range of level & $9.72-10.21$ & $8.63-9.13$ & $8.79-9.62$ & $8.78-9.37$ \\
\hline Change of phase & -0.45 & 0.5 & 0.28 & -0.14 \\
\hline Means of level & 10.03 & 8.91 & 9.25 & 9.13 \\
\hline Stability & $100.0 \%$ & $100.0 \%$ & $100.0 \%$ & $100.0 \%$ \\
\hline $\begin{array}{l}\text { Trend and } \\
\text { data path of trend }\end{array}$ & $\begin{array}{c}1 \\
(+)\end{array}$ & $\begin{array}{l}/ \\
(-)\end{array}$ & $\begin{array}{l}\text { / } \\
(-)\end{array}$ & $\begin{array}{c}\backslash \\
(+)\end{array}$ \\
\hline Stability of trend & $100 \%$ & $100 \%$ & $100 \%$ & $100 \%$ \\
\hline Interphase & \multicolumn{2}{|c|}{$\begin{array}{l}\text { Baseline phase/ } \\
\text { Intervention phase }\end{array}$} & $\begin{array}{l}\text { ntervention phase/ } \\
\text { Vithdrawal phase } 1\end{array}$ & $\begin{array}{l}\text { Withdrawal phase } 1 / \\
\text { Withdrawal phase } 2\end{array}$ \\
\hline \multicolumn{2}{|c|}{ Change between phases } & -1.09 & 0.21 & -0.25 \\
\hline \multicolumn{2}{|c|}{ Means between phases } & -1.12 & 0.34 & -0.12 \\
\hline \multicolumn{2}{|c|}{ Variability of trend and effect } & $\begin{array}{c}\text { V } \\
\text { Jegative }\end{array}$ & $\begin{array}{c}\| \\
\text { None }\end{array}$ & $\begin{array}{c}\text { V } \\
\text { Positive }\end{array}$ \\
\hline \multicolumn{2}{|c|}{$\begin{array}{l}\text { Variability of trend stability } \\
\text { Percentage of overlap }\end{array}$} & $\begin{array}{l}\text { le to stable } \\
0 \%\end{array}$ & $\begin{array}{c}\text { Stable to stable } \\
100 \%\end{array}$ & $\begin{array}{c}\text { Stable to stable } \\
100 \%\end{array}$ \\
\hline
\end{tabular}

\section{Discussion}

The results of 6MWT and TUG revealed that the physical performances of all the subjects were worse during the intervention phase than during the baseline phase, and then became much better during the first withdrawal phase. We supposed that the reason for this phenomenon is that the physical strength and endurance of the subjects diminished in the fitness training during the intervention phase. Compared with the other phases, the intervention phase is the most tiring, so the physical assessment score tends to decline. The second subject performed well in 6MWT during the withdrawal phases; both the first and second subjects had stable performance in TUG during the withdrawal phases, and the trends of those phases were positive. It meant that the physical training program was effective.

All of the three participants showed their will to join the somatosensory game for physical training, and none of them asked to take a rest when they were training with the rehabilitation system during the intervention phase. We speculate that, using a somatosensory game for rehabilitation training can enhance a patient's motivation to participate and maintain long-term exercise. Although one of the symptoms of schizophrenia includes the spreading of thoughts, the patients in this study who used this system for rehabilitation did not have a feeling of insecurity. This may be because the background image of the system was relatively simple or the subjects who were included in this study were relatively stable. This requires further study.

The limitations of this study were as follows: First, there were only seven types of rehabilitation movement in this rehabilitation system, which were insufficient for the patient who needs more training. Second, the same assessment was repeated multiple times for the same subject to collect multiple data. This may lead to the subject's boredom or practice effect on the assessment. This may also affect the level of intervention. Third, this study included only three subjects. We need more subjects to verify the results of the study. 


\section{Conclusions}

The key contributions of this study are as follows. First, this system is easy to operate even for patients with mental illness. Second, patients with schizophrenia are more willing to accept physical fitness training in the form of somatosensory games rather than traditional treatments. Third, the rehabilitation system using efficient vision-based action identification techniques is suitable for physical promotion programs for patients with schizophrenia. Fourth, the program in the rehabilitation system helps improve the physical performance of people with schizophrenia. Fifth, the system keeps a record of the rehabilitation processing and physical status as a treatment document of patients with schizophrenia.

\section{Acknowledgments}

This work was supported by the Ministry of Science and Technology of Taiwan under grant numbers MOST-106-2628-E-027-001-MY3 and MOST-106-2218-E-027-002.

\section{References}

1 M. Ding and G. Fan: IEEE Trans. Image Process. 25 (2016) 776. https://doi.org/10.1109/TIP.2015.2507445

2 M. Ding and G. Fan: Proc. IEEE Conf. Computer Vision and Pattern Recognition Workshops (IEEE, 2015$) 57$.

3 M. Ding and G. Fan: 2015 IEEE Winter Conf. Appl. Computer Vision (WACV) (IEEE, 2015) 47.

4 M. Ye, X. Wang, R. Yang, L. Ren, and M. Pollefeys: 2011 IEEE Int. Conf. Computer Vision (ICCV) (IEEE, 2011) 731.

5 A. Baak, M. Müller, G. Bharaj, H.-P. Seidel, and C. Theobalt: Consumer Depth Cameras for Computer Vision (Springer, London, 2013) p. 71. https://doi.org/10.1007/978-1-4471-4640-7_5

6 Z. Cao, T. Simon, S.-E. Wei, and Y. Sheikh: Proc. IEEE Conf. Computer Vision and Pattern Recognition (IEEE, 2017) 7291.

7 M. G. Jørgensen: Dan. Med. J. 61 (2014) B4775.

8 H. L. Bartlett, L. H. Ting, and J. T. Bingham: Gait \& Posture 39 (2014) 224. https://doi.org/10.1016/ j.gaitpost.2013.07.010

9 R. A. Clark, A. L. Bryant, Y. Pua, P. McCrory, K. Bennell, and M. Hunt: Gait \& Posture 31 (2010) 307.

10 S. Coodin: Can. J. Psychiatry 46 (2001) 549. https://doi.org/10.1177/070674370104600610

11 P. Gorczynski and G. Faulkner: Cochrane Database Syst. Rev. 5 (2010) CD004412.

12 D. Vancampfort, S. Rosenbaum, M. Probst, J. Connaughton, C. du Plessis, T. Yamamoto, and B. Stubbs: Disabil. Rehabil. 38 (2016) 2235. https://doi.org/10.3109/09638288.2015.1116622

13 J. A. DeLisa, B. M. Gans, and N. E. Walsh: Physical Medicine and Rehabilitation: Principles and Practice (Lippincott Williams \& Wilkins, Philadelphia, 2005) Vol. 1, p. 389.

14 M. H. Cameron and L. Monroe: Physical Rehabilitation for the Physical Therapist Assistant-E-Book (Elsevier Health Sciences, St. Louis, 2014) p. 506.

15 R. Taylor, A. Watt, H. Dalal, P. Evans, J. Campbell, K. Read, A. Mourant, J. Wingham, D. Thompson, and D. P. Gray: Int. J. Cardiol. 119 (2007) 196. https://doi.org/10.1016/j.ijcard.2006.11.018

16 J. Kvedar, M. J. Coye, and W. Everett: Health Aff. 33 (2014) 194. https://doi.org/10.1377/hlthaff.2013.0992

17 B. Lindberg, C. Nilsson, D. Zotterman, S. Söderberg, and L. Skär: Int. J. Telemed. Appl. 2013 (2013) 461829.

18 M. V. Bianciardi, S. Bella, F. Murgia, A. Carestia, and E. Prosseda: Clin. Ter. 167 (2016) e21.

19 R. Gattu, G. Teshome, and R. Lichenstein: Pediatr. Emerg. Care 32 (2016) 123.

20 B. L. Burke and R.W. Hall: Pediatrics 136 (2015) 293. https://doi.org/10.1542/peds.2015-1517

21 Y. L. Chen, C. H. Liu, and P. Lee: Appl. Sci. 8 (2018) 1161. https://doi.org/10.3390/app8071161

22 B. J. Byiers, J. Reichle, and F. J. Symons: Am. J. Speech-Lang. Pathol. 21 (2012) 397.

23 T. N. Tombaugh and N. J. McIntyre: J. Am. Geriatr. Soc. 40 (1992) 922.

24 R. A. Lasser, H. Nasrallah, L. Helldin, J. Peuskens, J. Kane, J. Docherty, and A. T. Tronco: Schizophr. Res. 96 (2007) 223.

25 A. P. Association: Diagnostic and Statistical Manual of Mental Disorders (American Psychiatric Association, Washington, DC, 2000) 4th ed., p. 309. 


$$
\text { 2018). }
$$

27 P. L. Enright: Respir. Care 48 (2003) 783.

28 T. Herman, N. Giladi, and J. M. Hausdorff: Gerontology 57 (2011) 203. https://doi.org/10.1159/000314963

29 J. C. Wall, C. Bell, S. Campbell, and J. Davis: J. Rehabil. Res. Dev. 37 (2000) 109.

30 D. Podsiadlo and S. Richardson: J. Am. Geriatr. Soc. 39 (1991) 142.

\section{About the Authors}

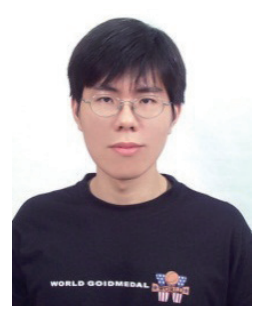

Yen-Lin Chen received his B.S. and Ph.D. degrees in electrical and control engineering from National Chiao Tung University, Hsinchu, Taiwan, in 2000 and 2006, respectively. He is now a professor at the Department of Computer Science and Information Engineering, National Taipei University of Technology, Taipei, Taiwan. Dr. Chen is a senior member of IEEE and a member of ACM, IAPR, and IEICE. In 2012, he earned the best annual paper award sponsored by the Intelligent Transportation Society of Taiwan. He also earned the Silver Invention Award from the Ministry of Economic Affairs of Taiwan and received the best annual ITS paper awards twice (2011 and 2012). His research interests include artificial intelligence, intelligent image analytics, embedded systems, pattern recognition, intelligent vehicles, and intelligent transportation systems. He has published over 100 journal and conference papers.

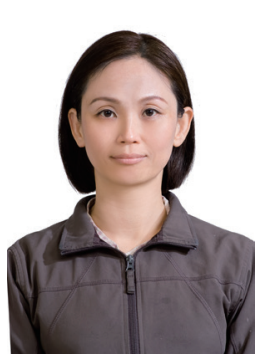

Chin-Hsuan Liu received her B.S. degree from Kaohsiung Medical University, Kaohsiung, Taiwan, in 2001 and M.Ed. degree from National Kaohsiung Normal University, Kaohsiung, Taiwan, in 2011. She is a Ph.D. candidate at the Department of Computer Science and Information Engineering, National Taipei University of Technology, Taipei, Taiwan. Her research interests are in schizophrenia, cognition, rehabilitation, and artificial intelligence.

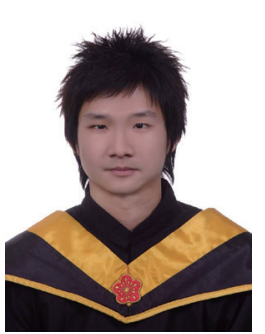

Chao-Wei Yu received his B.S. degree from Asia University, Taichung, Taiwan, in 2008 and M.A. degree from I-Shou University, Kaohsiung, Taiwan, in 2010. He received his Ph.D. degree from National Taipei University of Technology, Taipei, Taiwan, in 2017. His research interests are in embedded systems, IOT, intelligent image analytics, and cloud computing.

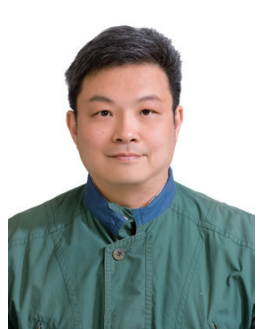

Posen Lee received his B.S. degree from Kaohsiung Medical University, Taiwan, in 1994 and his M.A. degree from New York University, USA, in 1998. He received his Ph.D. degree from National Kaohsiung Normal University, Taiwan, in 2012. Since 2013, he has been an assistant professor at I-Shou University. His research interests are in cognition, schizophrenia, and psychometrics. 